\title{
Motor Nuclear Representation of Masticatory Muscles in the Rat
}

\author{
Kazushige SASAmoto \\ Department of Physiology, Faculty of Dentistry, Kyushu University, \\ Fukuoka, 812 Japan
}

\begin{abstract}
Representation of the masticatory muscles within the motor trigeminal nucleus was studied in rats by the horseradish peroxidase (HRP) method and the antidromic field potential method. The motor trigeminal nucleus of the rat could be divided cytoarchitecturally into a dorsolateral and a ventromedial division. Within the dorsolateral division, the temporal muscle was represented dorsomedially, the masseter muscle dorsolaterally and laterally, and the lateral and medial pterygoid muscles ventrolaterally. Within the ventromedial division, the anterior digastric muscle was represented dorsomedially and the mylohyoid muscle ventrolaterally. Distribution of antidromic field potentials evoked by stimulation of the mylohyoid and masseteric nerves coincided with the results from the HRP investigation.
\end{abstract}

Representation of masticatory muscles in the motor trigeminal nucleus has been reported by earlier investigators using the degeneration method (SzENTÁGothai, 1949; Vedral and MatzKe, 1967). According to Szentágothai (1949), more dorsally situated masticatory muscles are represented more ventrally within the motor trigeminal nucleus. Recently, utilizing retrograde transport of horseradish peroxidase (HRP), the topographical localization of masticatory motoneurons has been reported (Mizuno et al., 1975; BATINI et al., 1976; MATSUdA et al., 1978). According to these recent results, dorsally situated jaw-closing muscles are represented more dorsally and laterally in the motor trigeminal nucleus than are the ventrally situated jaw-opening muscles. The aim of the present study was to ascertain in the rat the principle of myotopical arrangement of masticatory motoneurons within the motor trigeminal nucleus by the HRP method and by recording the antidromic potentials of motoneurons.

\section{METHODS}

Fifty-seven adult female rats (Wister King A), weighing 180 to $250 \mathrm{~g}$, were

Received for publication April 13, 1979

笹本一茂 
used.

HRP experiments. The animals were anesthetized with sodium pentobarbital (Somnopentyl, Pitman-Moore, Inc., $25 \mathrm{mg} / \mathrm{kg}$, i.p.). After exposing the temporal, masseter, mylohyoid, anterior digastric, lateral pterygoid or medial pterygoid muscle on the left side, a $10 \%$ solution of HRP (Sigma, type VI) in distilled water was injected into the belly of each muscle with a $100 \mu 1$ microsyringe calibrated in $1 \mu \mathrm{l}$ divisions. This was done very carefully to avoid the spreading of HRP out of the injected muscle. The animals in which any escape of HRP solution from the injected muscles was noted were discarded. The volume of injected HRP solution was 10 to $80 \mu \mathrm{l}$, depending on the size of the individual muscle. After administration of HRP, the incisions in the skin were sutured, and the animals were allowed to survive for 24 to $48 \mathrm{hr}$.

After the survival period, the animals were deeply anesthetized with sodium pentobarbital $(50 \mathrm{mg} / \mathrm{kg}$, i.p.) and perfused through the left cardiac ventricle successively with $40 \mathrm{ml}$ of saline and $40 \mathrm{ml}$ of a fixative composed of $1 \%$ paraformaldehyde and 2.5\% glutaraldehyde (TAAB) in Millonig's buffer ( $\mathrm{pH} 7.3$ ). The brains were removed promptly, postfixed in a fresh prepared fixative of the same composition as above for 4 to $6 \mathrm{hr}$ at $4{ }^{\circ} \mathrm{C}$ and then kept in Millonig's buffer over night. Transverse serial sections of the brainstem were made at $60 \mu \mathrm{m}$ thickness with a Vibratome (Oxford) floating in a $0.05 \mathrm{~m}$ Tris-HCl buffer ( $\mathrm{pH} 7.6)$. The sections were treated with $0.05 \%$ 3,3'-diaminobenzidine tetrahydrochloride (Merck) and $0.01 \%$ hydrogen peroxide in $0.05 \mathrm{M}$ Tris- $\mathrm{HCl}$ buffer at room temperature for 15 to 30 min (GRAHAM and KARNOVSKY, 1966). The sections were counterstained with $0.1 \%$ thionine, dehydrated, cleared and then mounted on slides.

Electrophysiological experiment. The animals were anesthetized with urethan $(1 \mathrm{~g} / \mathrm{kg}$, i.p.). The mylohyoid nerve, supplying the anterior digastric and mylohyoid muscles, and the masseter nerve were prepared for electrical stimulation on the left side. Bipolar silver wire electrodes (interpolar distance: $1.5 \mathrm{~mm}$ ), insulated with acrylic resin except for the tips, were used to stimulate the nerves. The electrodes were sutured with the muscles and the skin. The animal's head was fixed in a stereotaxic apparatus (Narishige) according to the method of AlBEFESSARD et al. (1966). A small hole for inserting a recording electrode into the motor trigeminal nucleus was made in the occipital bone. Body temperature was maintained at $37-38^{\circ} \mathrm{C}$ throughout the experiment.

Stimuli applied to the muscle nerves were square waves of $0.05 \mathrm{msec}$ duration and 0.2 to $2.0 \mathrm{~mA}$ intensity. Potentials evoked in the motor trigeminal nucleus by antidromic stimulation of peripheral nerves were recorded extracellularly with a glass-coated tungsten electrode (DC resistance, 2-5 M 2 ) or a glass microelectrode filled with $3 \mathrm{M} \mathrm{KCl} \mathrm{(3-10} \mathrm{M} \Omega$ ). The recording electrodes were carried by a motordriven manipulator (Narishige). The position of the recording electrode was measured antero-posteriorly from the lambda, laterally from the midline and verti- 


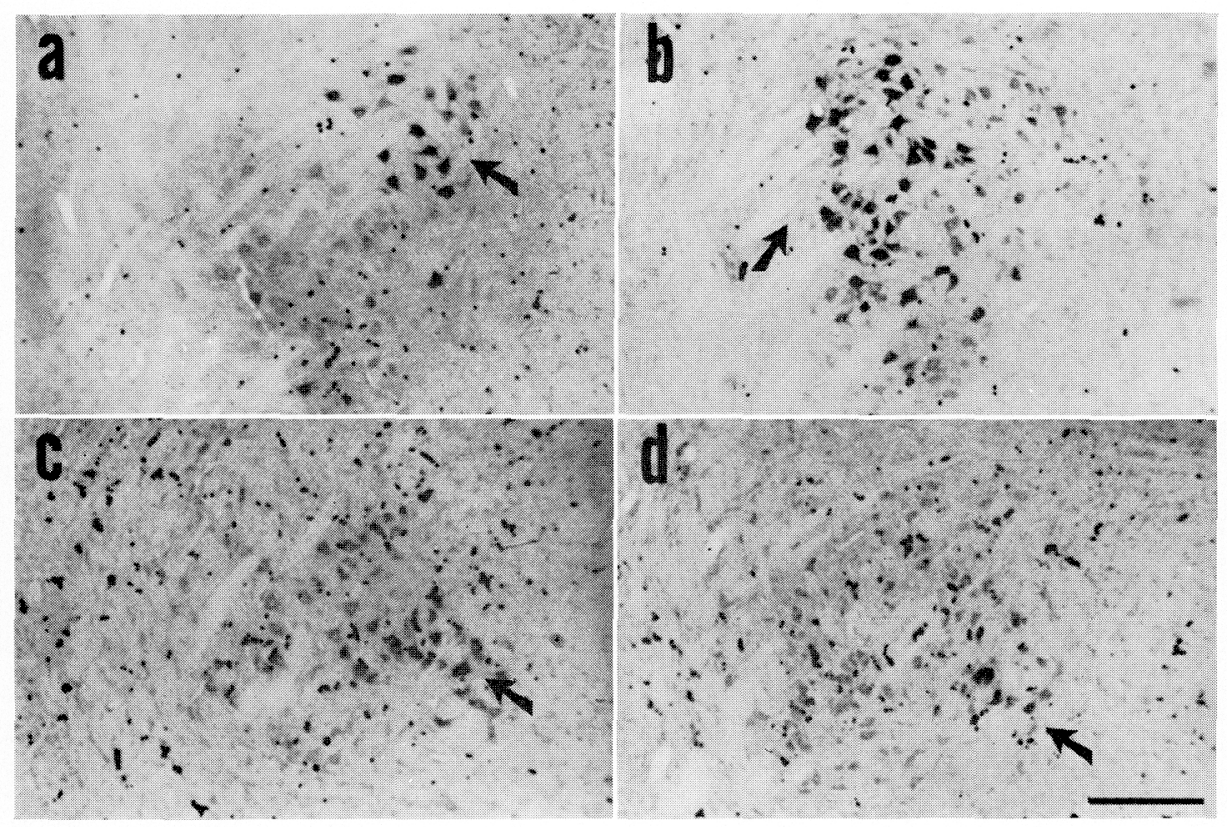

Fig. 1. Frontal sections through the motor trigeminal nucleus. Darkly stained HRPlabelled masticatory motoneurons are indicated by arrow; temporal (a) and masseter (b) motoneurons in the rostral third, and mylohyoid (c) and anterior digastric (d) motoneurons in the caudal third. The ventromedial division is seen in the caudal third but not in the rostral third. Dorsal, up and medial, right. Horizontal bar, $300 \mu \mathrm{m}$.

cally from the cortical surface.

The evoked potentials were amplified and displayed on the screen of a cathode ray oscilloscope (Nihon Kohden, VC 7). After the experiment, a DC current $(10 \mu \mathrm{A}$ for $10 \mathrm{sec})$ was passed through the recording electrode at the point of maximum response in the motor trigeminal nucleus to make a small lesion. The animal was then sacrificed and perfused with $10 \%$ formalin through the left cardiac ventricle. The excised brain was fixed in $10 \%$ formalin. Serial $60 \mu \mathrm{m}$ sections were made and stained with thionine to locate the recording site.

\section{RESULTS}

\section{HRP investigation of nuclear representation of masticatory muscles}

As described by Mizuno et al. (1975), the rat motor trigeminal nucleus could be divided cytoarchitecturally into two divisions: a large dorsolateral division and a small ventromedial division. The dorsolateral division was seen through the entire rostrocaudal extent of the nucleus, while the ventromedial division was observed only at levels of the caudal half of the nucleus (Fig. 1).

After injection of the HRP solution into each masticatory muscle (in seven 

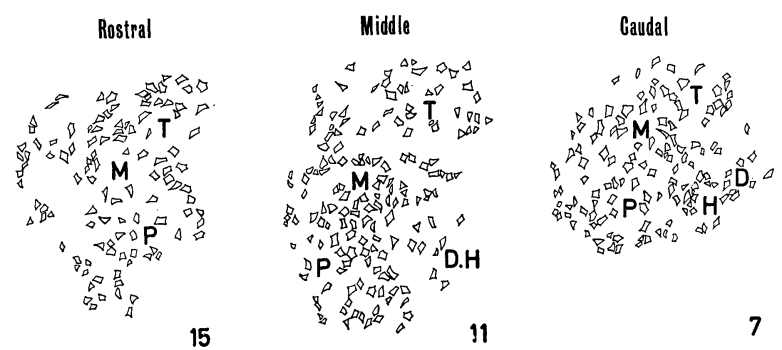

Fig. 2. Representation of masticatory muscles in the rostral, middle and caudal thirds of the motor trigeminal nucleus. Number indicates the position of serial $60 \mu \mathrm{m}$ sections from the caudal (1) to rostral end (20) of the motor trigeminal nucleus. T, M, P, D, and $\mathrm{H}$ represent temporal, masseter, pterygoid, anterior digastric and mylohyoid muscles, respectively.

animals), HRP-labelled motoneurons could be identified by the presence of the brown granules of HRP reaction products in their somata and proximal dendrites. These labelled motoneurons were aggregated in a cluster within the ipsilateral motor trigeminal nucleus and no labelled motoneurons were found contralaterally. Following injection of HRP into the temporal, masseter, lateral pterygoid or medial pterygoid muscles, nerve cells labelled with HRP were found in the dorsolateral division of the nucleus; temporal motoneurons were located dorsomedially (Fig. 1a), masseter motoneurons dorsolaterally and laterally (Fig. 1b) and pterygoid motoneurons ventrolaterally. In the ventromedial division, motoneurons were labelled with the HRP that was injected into the mylohyoid or anterior digastric muscle. Mylohyoid motoneurons were observed in the ventrolateral part of the ventromedial division (Fig. 1c) and anterior digastric motoneurons were in the dorsomedial part of the ventromedial division (Fig. 1d). These results are summarized diagramatically in Fig. 2.

Some small neurons in the ventral aspect of the dorsolateral division at the middle level of the motor trigeminal nucleus were found free from HRP-labelling in all animals used in the present study. These small neurons were assumed to be motoneurons supplying the tensor tympani and tensor veli palatini muscles (MrzuNo et al., 1975). These muscles were not injected with HRP in the present study.

In two animals, a small number of trigeminal mesencephalic neurons at the level of the motor trigeminal nucleus were found labelled with HRP that was injected into the masseter muscle.

Distribution of antidromic responses evoked by stimulation of the masseteric and mylohyoid nerves

Electrical stimulation of the masseteric or mylohyoid nerve evoked field potentials in the motor trigeminal nucleus. In each case, the evoked potential was usually triphasic, starting with the positive deflection and showing the largest amplitude in the negative deflection. The entire duration of the evoked potential was about 

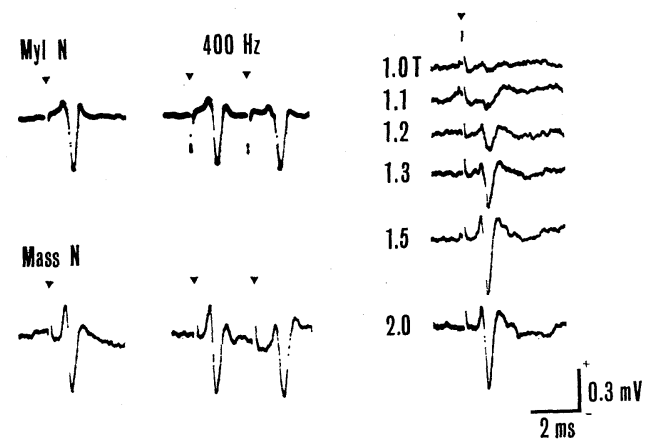

Fig. 3. Antidromic field potentials recorded in the motor trigeminal nucleus. Left: antidromic potentials evoked by single pulse stimulation of mylohyoid nerve (Myl N) and masseteric nerve (Mass $\mathrm{N}$ ). Middle: antidromic potentials faithfully followed repetitive stimulation at $400 \mathrm{~Hz}$. Right: antidromic potentials evoked by varying strengths of stimulation of masseteric nerve. $T$ indicates threshold intensity $(0.25 \mathrm{~mA})$. Triangle indicates shock artifacts. Polarity, positivity upward.

$1.5 \mathrm{msec}$ or less. Their latencies were short and constant; the mean peak latency of the masseteric response was $0.56 \mathrm{msec}$ and that of the mylohyoid response was $0.60 \mathrm{msec}$. They followed repetitive stimulation at frequencies exceeding $400 \mathrm{~Hz}$. The amplitude increased with the increasing intensity of nerve stimulation; the maximum amplitude was reached by an intensity 1.5 times the threshold level (Fig. 3). It is thus concluded that they were antidromic field potentials. When the frequency of repetitive stimulation exceeded $800 \mathrm{~Hz}$, a step appeared in the rising phase of the potential and the amplitude was reduced (not shown in the figure). This was interpreted as being due to blocking of the soma-dendritic potential at the initial segment in motoneurons (LANDGREN and OLSSON, 1976).

Figure 4 shows how the amplitude of the negative deflection of the antidromic response changed along the antero-posterior $(P)$, medio-lateral $(L)$ and vertical $(D)$ directions. The data were obtained from one animal. The mylohyoid potential was composed of antidromic excitation of mylohyoid-digastric motoneurons evoked by stimulation of the mylohyoid nerve innervating both the mylohyoid and anterior digastric muscles. The coordinates of points of the maximum massetoric or mylohyoid response averaged in five cases are shown in Table 1. As shown in Fig. 4 and Table 1, the site of the maximum mylohyoid-digastric

Table 1. Coordinates (mean $\pm \mathrm{SE}$ ) of the recording points for antidromic masseteric (Mass) and mylohyoid (Myl) responses.

\begin{tabular}{lccc}
\hline & $P(\mathrm{~mm})$ & $L$ & $D$ \\
\hline Mass & $0.64 \pm 0.09$ & $2.06 \pm 0.06$ & $6.90 \pm 0.10$ \\
Myl & $0.96 \pm 0.07$ & $1.68 \pm 0.05$ & $7.46 \pm 0.09$ \\
\hline
\end{tabular}

$P$, antero-posterior distance from lambda; $L$, medio-lateral distance from midline;

$D$, depth from cortical surface. Number of animals: 5 . 

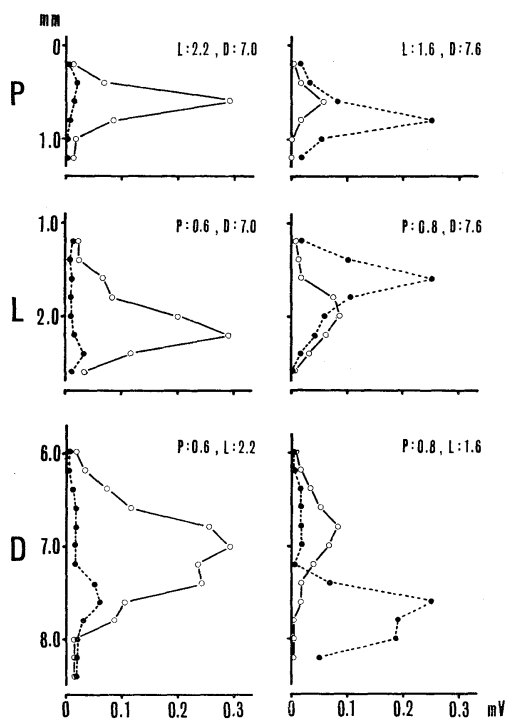

Fig. 4. Spatial distribution of antidromic response of masseteric (open circles) and mylohyoid neurons (closed circles). Amplitudes of the negative component of the antidromic response (abscissae) were plotted against distances in the antero-posterior $(P)$, medio-lateral $(L)$ and vertical $(D)$ directions. They were measured from the lambda, midline and cortical surface, respectively. In each diagram, recordings were made by advancing the electrode in only one direction. The fixed values of coordinates are indicated in the upper right of each diagram.

response was located approximately $0.3 \mathrm{~mm}$ caudally, $0.4 \mathrm{~mm}$ medially and $0.5 \mathrm{~mm}$ ventrally from that of the masseteric response, and these sites were histologically verified to exist within the motor trigeminal nucleus. The diameters of the motor trigeminal nucleus measured in histological preparations were 634 115 (mean \pm SE, $n=5) \mu \mathrm{m}$ medio-laterally, $926 \pm 19 \mu \mathrm{m}$ dorso-ventrally and $1,230 \pm 52 \mu \mathrm{m}$ rostro-caudally. Assuming the shrinkage of the preparation due to our histological procedure to be $20 \%$, the actual diameters of the motor trigeminal nucleus would be about $800 \mu \mathrm{m}, 1,200 \mu \mathrm{m}$ and $1,500 \mu \mathrm{m}$, respectively. The distribution of mylohyoid-digastric responses and that of masseteric responses examined by the antidromic field potential method coincided well with the results from the HRP method, showing that the center of the mylohyoid-digastric motoneuron group was located about $0.3 \mathrm{~mm}$ caudally, $0.4 \mathrm{~mm}$ medially and $0.4 \mathrm{~mm}$ ventrally from that of masseteric motoneuron group.

\section{DISCUSSION}

The motor nuclear representation of the masticatory muscles as determined by the HRP method. The motor trigeminal nucleus of the rat can be divided 
cytoarchitecturally into dorsolateral and ventromedial divisions, as has been described in the cat by TABER (1961), and in the cat and rat by MizunO et al. (1975). Within the dorsolateral division of the motor trigeminal nucleus, the temporal muscle was represented dorsomedially, the masseter muscle dorsolaterally and laterally, and the pterygoid muscles ventrolaterally. Within the ventromedial division of the motor trigeminal nucleus, the anterior digastric muscle was represented in the dorsomedial part, and the mylohyoid muscle in the ventrolateral part.

The location of masticatory motoneurons in the motor trigeminal nucleus has been studied by several investigators. Szentágothai (1949) made lesions in the motor trigeminal nucleus of the cat stereotaxically and examined degenerated motor nerve fibers and motor end-plates. VeDRAL and MATzKe (1967) transected the motor nerves to the masticatory muscles in the cat and detected chromatolytic neurons. According to these authors, dorsally situated masticatory muscles were represented ventrally within the motor trigeminal nucleus. The present study in rats led to conclusions which differed from those obtained by the degeneration methods, and supported the recent HRP studies performed in rats (LimwongSE and DeSAntis, 1977; DeSantis et al., 1978), cats (Mizuno et al., 1975; Batini et al., 1976) and rabbit (MATSUDA et al., 1978). YASSIN and LEONG (1979) showed by the HRP method that the temporal muscle in the rat and monkey was represented dorsomedially, ventromedially and ventrolaterally in the motor trigeminal nucleus. On the other hand, Mrzuno et al. (1975) showed that the temporal muscle in the rat and cat was represented dorsally and dorsomedially. Still other HRP studies demonstrated a dorsal representation of the temporal muscle in the nucleus (BATINI et al., 1976; LimwongSe and DeSANTis, 1977; MATSuda et al., 1978). In the present study, the temporal motoneurons in the rat were distributed in the dorsomedial portion but not in the ventral portion of the motor trigeminal nucleus. According to BATINI et al. (1976), anterior digastric and mylohyoid motoneurons were distributed throughout the entire rostrocaudal extent of the motor trigeminal nucleus in cats, but Mizuno et al. (1975) and Limwongse and DeSAntis (1977) found that they were located only in the caudal half of the motor trigeminal nucleus in cats and rats. MATSUdA et al. (1978) also arrived at the same conclusion for rabbits. The present findings support those of Mizuno et al. (1975), LimwongSE and DeSAntis (1977) and MATSuda et al. (1978). The motoneurons of anterior digastric and mylohyoid muscles were located in a small ventromedial division which was found in the caudal half of the motor trigeminal nucleus. Thus, motoneurons innervating jaw-closing muscles are distributed more dorsolaterally or laterally than those innervating jaw-opening muscles.

The tensor tympani and tensor veli palatini muscles were not injected with HRP in the present study. The small motoneurons distributed in the ventral portion of the dorsolateral division of the motor trigeminal nucleus at the middle levels were never labelled with HRP in any of the animals examined in the present 
study. According to Szentágothai (1949), the motoneurons supplying these muscles are located in the ventral portion of the motor trigeminal nucleus. These small neurons were thus assumed to be motoneurons innervating the tensor tympani and the tensor veli palatini muscles, as was also suggested by Mizuno et al. (1975).

In the present study, a few neurons in the trigeminal mesencephalic nucleus were labelled with HRP injected into the masseter muscle. Similar observations were made by Mizuno et al. (1975) and Limwongse and DeSAntis (1977). Accumulation of HRP reaction products in mesencephalic neurons was considered to be due to uptake and anterograde transport in the muscle spindle afferent fibers from the masseter muscle, since such transport has been demonstrated in trigeminal ganglion cells (FURSTMAN et al., 1975), and the trigeminal mesencephalic nucleus has been known to contain cell bodies of muscle spindle afferent neurons supplying the masseter muscle (CORBIN, 1940; SzentáGOTHAI, 1948; JERGE, 1963; KuBota et al., 1978).

Distribution of antidromic field potentials of the mylohyoid-digastric and masseteric motoneurons. A principal aim of this experiment was to correlate the results of the antidromic field potential method with those of the HRP method. The positions of neurons innervating the mylohyoid and anterior digastric muscles and the masseter muscle determined by antidromic stimulation were well within the dimensions of the motor trigeminal nucleus. LANDGREN and OLSSON (1976) mapped extracellular focal potentials evoked by antidromic stimulation of the anterior digastric or masseter nerve. They found that the focus of the digastric antidromic potential was medially in the caudal third, and ventromedially in the middle third of the motor trigeminal nucleus, and that of the masseteric response was laterally in the middle and rostral third of the nucleus. DeSANTIS et al. (1978) recorded antidromic field potentials in the motor trigeminal nucleus of rats that received HRP injections into either the masseter or the mylohyoid and anterior digastric muscles. They found that the masseter motoneurons were located dorsally and laterally to those innervating the mylohyoid and anterior digastric muscles. The foci of mylohyoid-digastric and masseteric responses determined in the present study coincided with those described by LANDGREN and OLSSON (1976) and DeSantis et al. (1978). It was, thus, definitely shown that the antidromic field potential maxima occurred very near the center of the neuron distribution determined by the HRP method.

The author thanks Professors K. Hasegawa, H. Kuriyama and M. Ohta for their encouragement and advice during this work. Thanks are also due to Dr. A. Simpson for his critical reading of the manuscript and his suggestions. The author is grateful to Miss N. Shirakami and Miss Y. Kikuchi for their valuable help in preparing the manuscript.

\section{REFERENCES}

Albe-Fessard, D., Stutinsky, F., and Libouban, S. (1966) Atlas Stéréotaxique du Diencéphale 
du Rat Blanc, Presses de 1'Imprimerie Prister, Paris.

Batini, C., Buisseret-Delmas, C., and Corvisier, J. (1976) Horseradish peroxidase localization of masticatory muscle motoneurons in cat. J. Physiol. (Paris), 72: 301-309.

Corbin, K. B. (1940) Observations on the peripheral distribution of fibers arising in the mesencephalic nucleus of the fifth cranial nerve. J. Comp. Neurol., 73: 153-177.

DeSantis, M., Limwongse, V., and Rigamonti, D. (1978) Somatotopy in the trigeminal motor nucleus of the rat: Field potentials recorded in the neuron pool after retrograde transport of horseradish peroxidase. Neurosci. Lett., 10:95-98.

Furstman, L., Saporta, S., and Kruger, L. (1975) Retrograde axonal transport of horseradish peroxidase in sensory nerves and ganglion cells of the rat. Brain Res., 84: 320-324.

GraHAM, R. C. and KARNovsky, M. J. (1966) The early stages of absorption of injected horseradish peroxidase in the proximal tubules of mouse kidney: Ultrastructural cytochemistry by a new technique. J. Histochem. Cytochem., 14: 291-302.

JERGE, C. R. (1963) Organization and function of the trigeminal mesencephalic nucleus. $J$. Neurophysiol., 26: 376-392.

Kubota, K., Masegi, T., and SAto, Y. (1978) Evolutionary outlook of the masticatory proprioceptive innervation involved in mammalian jaw movements. J. Stomatol. Soc. Jpn., 45: 1-19 (in Japanese).

LANDGRen, S. and Olsson, K. A. (1976) Localization of evoked potentials in the digastric, masseteric, supra- and intertrigeminal subnuclei of the cat. Exp. Brain Res., 26: 299-318.

Limwongse, V. and DeSANTis, M. (1977) Cell body locations and axonal pathways of neurons innervating muscles of mastication in the rat. Am. J. Anat., 149: 477-488.

Matsuda, K., Uemura, M., Kume, M., Matsushima, R., and Mizuno, N. (1978) Topographical representation of masticatory muscles in the motor trigeminal nucleus in the rabbit: A HRP study. Neurosci. Lett., 8: 1-4.

Mizuno, N., Konishi, A., and SATo, M. (1975) Localization of masticatory motoneurons in the cat and rat by means of retrograde axonal transport of horseradish peroxidase. $J$. Comp. Neurol., 164: 105-116.

Szentágothai, J. (1948) Anatomical consideration of monosynaptic reflex arcs. J. Neurophysiol., 11: 445-454.

Szentágothai, J. (1949) Functional representation in the motor trigeminal nucleus. J. Comp. Neurol., 90: 111-120.

TABER, E. (1961) The cytoarchitecture of the brain stem of the cat. I. Brain stem nuclei of cat. J. Comp. Neurol., 116: 27-70.

Vedral, D. F. and Matzke, H. A. (1967) Topographical localization of the muscles of mastication in the motor nucleus of the trigeminal nerve in the cat. J. Hirnforsch., 9: 565-569.

YASsIN, I. B. H. M. and LeONG, S. K. (1979) Localization of neurons supplying the temporalis muscle in the rat and monkey. Neurosci. Lett., 11: 63-68.

Vol. 29, No. 6, 1979 